\title{
Centralized Protection Strategy for Medium Voltage DC Microgrids
}

\author{
Mehdi Monadi, Member, IEEE, Catalin Gavriluta, Student Member, IEEE, Alvaro Luna, Member, \\ IEEE, Jose Ignacio Candela, Member, IEEE, Pedro Rodriguez, Fellow, IEEE
}

\begin{abstract}
This paper presents a centralized protection strategy for medium voltage dc (MVDC) microgrids. The proposed strategy consists of a communication-assisted fault detection method with a centralized protection coordinator and a fault isolation technique that provides an economic, fast, and selective protection by using the minimum number of $\mathrm{dc}$ circuit breakers (DCCBs). The proposed method is also supported by a backup protection which is activated if communication fails. The paper also introduces a centralized self-healing strategy that guarantees successful operation of zones that are separated from the main grid after the operation of the protection devices. Furthermore, to provide a more reliable protection, thresholds of the protection devices are adapted according to the operational modes of the microgrid and the status of distributed generators (DGs). The effectiveness of the proposed protection strategy is validated through real-time simulation studies based on the hardware in the loop (HIL) approach.
\end{abstract}

Index Terms - Adaptive protection, centralized protection, smart dc microgrids.

\section{INTRODUCTION}

Due to the increasing penetration of DGs, especially in the form of renewable energy systems (RES), the concept of microgrids has been proposed as a method for DG integration into the electrical grids. Microgrid is a common concept in both ac and dc systems and is defined as a smallscale low or medium voltage grid consisting of loads and DGs. Such a system is capable of operating in both islanded and grid-connected modes [1]. Because of the advantages of the dc networks over the ac grids, and also because of the new developments in the technology of voltage source converters (VSCs), nowadays there is a major interest in dc grids in both research and industrial realms [2-5].

At the present moment, protection is one of the most important challenges in the development of dc microgrids. Protection issues mainly arise due to the particular behavior of the fault current in VSC-based networks [6]. When a fault occurs in a dc grid, firstly, the dc-link capacitor is discharged causing the voltage of the main dc bus to drop precipitously. Then, the energy stored in the cable

This work was supported in part by the Spanish Ministry of Economy and Competitiveness under Project ENE2013-48428-C2- 2-R. The work of M. Monadi was supported by the Ministry of Science, Research, and Technology, Iran.

M. Monadi is with Technical University of Catalonia (UPC) Barcelona, Spain and Shahid Chamran University of Ahvaz, Ahvaz, Iran (e-mail: meh_monadi@yahoo.com).

C. Gavriluta is with the Grenoble Electrical Engineering Laboratory (G2ELab), France (email: catalin.gavriluta@g2elab.grenoble-inp.fr).

A. Luna, J. I. Candela are with Technical University of Catalonia (UPC) Barcelona, Spain. (e-mails: luna@ee.upc.edu, candela@ee.upc.edu)

P. Rodriguez is with Technical University of Catalonia (UPC) Barcelona, Spain and Abengoa research, Sevilla, Spain (e-mail: prodriguez@ee.upc.edu). inductance is also discharged through the freewheeling diodes of the VSCs. Subsequent to the fault occurrence, the control scheme of the converter turns off the main switches of the VSC (e.g. IGBTs) to protect them against the overcurrent; hence, the VSC operates as an uncontrolled full-bridge rectifier and the fault will be fed from the ac side of the VSC (through the freewheeling diodes paths) [7]. Therefore, fault currents in VSC-based dc networks will have three different components, each with its special characteristics: i) the dc link capacitors discharge current, ii) the cable inductance discharge through the freewheeling diodes, and iii) the ac-grid current [8]. Given this fault current behavior, the conventional protection devices and methods that are used in ac systems are faced with new challenges. For example, the electromechanical circuit breakers $(\mathrm{CBs})$ that are used in ac networks are not fast enough to protect the vital and vulnerable components of the VSCs against the faults in dc networks [9]. This is due to the fact that an adequate protection scheme for the $\mathrm{dc}$ microgrids should be able to operate during the capacitor discharge period to prevent the fault current from flowing through the VSC components. In other words, the critical operating time for the protection of a VSC is the beginning of the second component of the dc fault current and before the fault current starts flowing through the freewheeling diodes. As this time is given by the size of the dc-link capacitor, it will be typically very short (in the range of a few milliseconds) [8]. Hence, a relatively faster protection device is required for de networks [10]. Different types of DCCBs can be found; however, they are more expensive than their ac counterparts, especially in the level of medium voltage. Therefore, it is not economically feasible to use individual DCCBs for all the feeders of a microgrid.

Another important aspect is that, although overcurrent relays (OCRs) can provide fast fault detection and fast tripping, coordination of theses relays and providing a selective protection is a challenging task in VSC-based dc systems. This is mainly due to the reason that the dc line reactance is fairly lower than the counterpart ac systems. Therefore, considering the small length of distribution lines, it is difficult to distinguish between faults that occur inside or outside of a protected line [11]. In addition, because of the very fast increment in the dc fault current, it is very difficult to coordinate the conventional time-inverse OCRs in dc networks. Also, the performance analysis of the OCRs operating in dc networks shown in [7] illustrates that there is not enough time interval between the operation of series OCRs to guarantee their coordinated operation. Moreover, the connection of a DG to a distribution feeder can change the power flow and the fault current direction; this may disturb the coordination of the consecutive OCRs.

For these reasons, the protection of dc microgrids requires 
faster, more reliable and more accurate methods. Moreover, the necessity of a relatively faster protection scheme makes it impossible to obtain useful data for fault location from the voltage and current waveforms [7, 12]. This aspect also makes it difficult to use fault detection methods with time consuming calculations.

Compared to conventional protection strategies, communication-assisted methods can provide a fast and selective approach without using any complex and time consuming algorithms. By use of communication links, the differential method can be implemented in the dc feeders. The differential protection is able to detect the exact faulty part of the dc network, considering the high rising rate of the dc fault current [13]. Also, the connection/disconnection of DGs cannot disturb the performance of differential-based methods. Furthermore, this method is more sensitive than overcurrent relays and is able to detect high impedance faults (HIFs) [1] .

To address some of the presented challenges regarding protection in dc microgrids a communication-assisted technique is proposed in this paper. The proposed protection strategy consists of: 1) a differential based relay which is used for fault location and detection inside sub-microgrids; 2) an overcurrent-based relay to protect the VSCs connected to the host network and the DGs; 3 ) a centralized protection unit (CPU) which supervises the protection devices and adapts them with the operational conditions of the microgrid. This unit also executes a self-healing process to guarantee the supply-adequacy of the islanded microgrids and on-outage sub-microgrids; 4) a combination of DCCBs and isolators to provide a fast and selective fault interruption with the minimum number of DCCBs. All the components of the proposed protection and self-healing strategy are coordinated together. In the first step, after the fault detection and location by the proposed relays, since the common VSCs are not able to block the fault current, all the corresponding DCCBs will operate and interrupt the fault. Afterwards, the fault will be isolated by the associated isolators. Finally, after the network restoration, the proposed self-healing method will be executed in order to guarantee the stable operation of the isolated zones of the microgrid.

The proposed technique is also complemented by a backup protection mechanism which is activated when the communication link fails. Moreover, the hardware-in-theloop (HIL) approach is used to evaluate the performance of the proposed strategy. Using this method we take into consideration errors and delays that do not appear in off-line simulation, such as time delay in data transfer.

The paper is organized as follows: Section II describes the dc microgrid that will be used as study-case for the paper. The components and the steps of the proposed protection are explained in Section III and IV, respectively. The operation of the proposed self-healing and adapting (SHA) unit is explained in Section V. The HIL setup is introduced in Section VI. Finally, in Section VII the performance of the proposed scheme is illustrated for some case studies.

\section{DC-MICROGRID STUDY-CASE}

The performance of the proposed protection technique is explained and evaluated, for a set of case studies in the context of a hypothetical dc distribution network, as shown in Fig. 1. The basic configuration and parameters of this network are extracted from the benchmark proposed in [14] and re-designed to operate as a dc microgrid. The network is a VSC-based dc system consisting of: DGs which are interfaced to the grid through VSCs, residential/industrial loads, and the dc feeders.

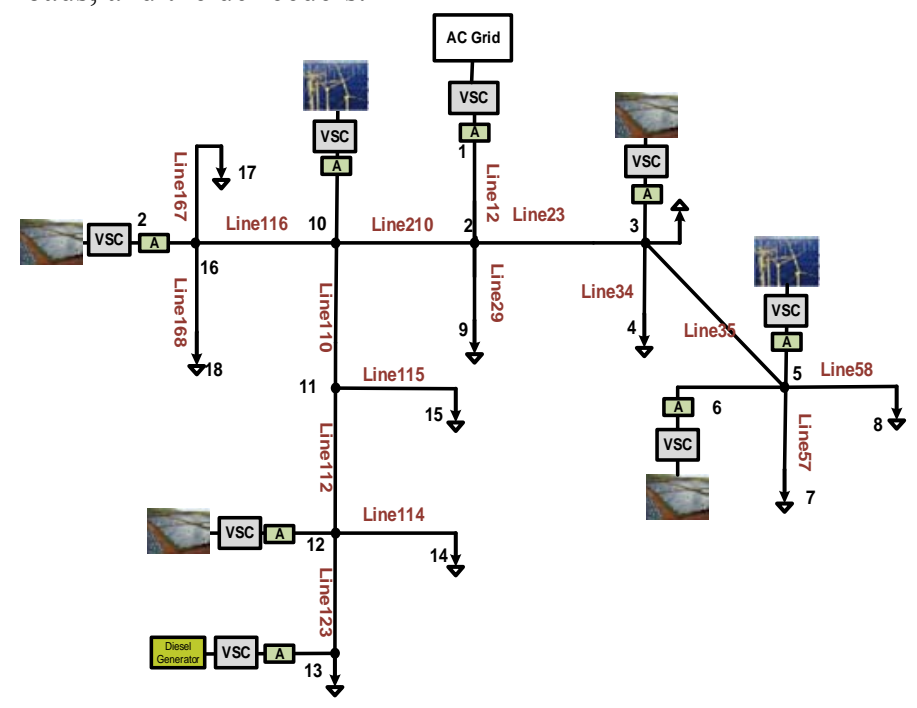

Fig. 1. DC microgrid study case.

Two-level $\pm 10-\mathrm{kV}$ VSCs are used to interface the connection to the ac network as well as the RESs to the dc network. Hall-Effect current transducers (CTs) are installed on both ends of the dc lines. The data transfer amongst the $\mathrm{dc}$ buses and the centralized protection unit is made possible via dedicated communication links.

\section{COMPONENTS OF THE PROPOSED PROTECTION} SCHEME

Protection systems must be able to detect and locate the various types of faults, interrupt the fault current and isolate the faulty zones before serious damage is inflicted on vital devices. The following subsections describe the method and devices that are used in the proposed strategy to achieve the protection goals.

\section{A. Fault Interruption and Isolation}

Since common VSCs are not capable of fault-blocking, the fault current should be interrupted by the appropriate DCCBs. However, due to the specification of these CBs, they are more expensive than their counterpart ac breakers. Therefore, installing DCCBs in all the dc lines, although it provides a selective and reliable protection, increases substantially the initial cost of the protection scheme. Hence it might not be applicable for most dc distribution systems and microgrids. Therefore, in this paper, a combination of DCCBs and isolator switches are used to provide an economic and selective protection scheme.

After a fault event, the fault is fed by the sources which are connected to the microgrid. Of course, the contribution of each source in the fault current is related to different factors such as the source location and its capacity. As shown in Fig. 1, VSCs are located in the microgrid at the point of connection to the host network as well as at the different connection points of the various DGs. Thus, in order to interrupt the fault current and to protect the VSCs and the network elements, it is necessary to install DCCBs at the connection point of the microgrid to the host network 
and at the connection point of DGs, i.e., the points labeled "A" in Fig. 1. Solid-state CBs (SSCBs) are the fastest type of DCCBs that can operate in less than $1 \mathrm{~ms}[15,16]$; this type of DCCBs are considered in the paper.

On the other hand, in order to provide a selective protection, the faulty line/busbar should be isolated and the rest of the network should continue its normal operation. This can be achieved by installing the appropriate isolator switches on both ends of the main dc lines. These switches are cheaper than DCCBs; however, they must be opened/closed only in the no-load condition. Therefore, they isolate the faulty part only after the fault current was interrupted by the DCCBs.

\section{B. Forming Sub-microgrids (SMGs)}

The use of a combination of DCCBs and isolators results in a "cut and try" process. During this process, the entire microgrid is disconnected from the sources and, after the fault is isolated, the rest of the network is reconnected again. In the proposed protection scheme, to prevent the overall outage and to limit the negative effects of the cut and try process, the microgrid is clustered into several submicrogrids. The sub-microgrids are connected together using DCCBs. In other words, the boundaries of the submicrogrids are determined by the location of the DCCBs. By use of this clustering, the cut-and-try process is done only for the faulted sub-microgrid. It has to be noted that determining the optimal placement of these CBs (i.e., optimal microgrid clustering) is out of the scope of this paper; however, as mentioned in [17] factors such as supply-adequacy can be considered to facilitate the operation of the sub-microgrids even if they are disconnected from the rest of the network. These DCCBs also play an important role in the backup protection which is executed when the communication link fails, as it will be explained in Section IV.B.

\section{Fault Detection and Location}

As mentioned above, the fault current is supplied through the VSC stations of the host grid and of the DGs. These VSCs are vulnerable against faults on their dc side; hence, fast fault detection is necessary for the VSC stations. This can be achieved by current monitoring at the connection points of the VSCs and microgrid, i.e., points labeled " $A$ " in Fig. 1. For this reason, VSC stations are equipped with an overcurrent-based relay labeled as source protection relays (SPR). The first stage of the fault current, i.e., capacitor discharge current, has a high increasing rate. Hence, the SPRs can detect the fault after several microseconds. Settings of these relays are determined based on the critical time of the corresponding VSCs and the load current. Other specifications and tasks of the SPRs are explained in Section IV.

Monitoring the currents at the "A" points however, does not determine the exact location of the fault and cannot provide a selective protection. Moreover, in the case of HIF occurrence, the SPR may not even detect the fault. For these reasons, in the proposed strategy, the fault location is handled by the current-differential-based method. The differential relays are more accurate than OCR and are able to identify the exact faulty line/busbar [1, 18]. Moreover, unlike the time-inverse OCR, their performance is not impacted by the high rising rate of the dc fault current. Therefore, although differential-based methods need communication links and more current transducers than the OC-based methods, they can provide a fast and selective protection that is not affected by the intermittent behavior of DGs. It should be pointed out that the communication infrastructure and capabilities of the smart grids could be used for implementing the differential-based methods. By using this infrastructure no additional costs are required for constructing new communication links.

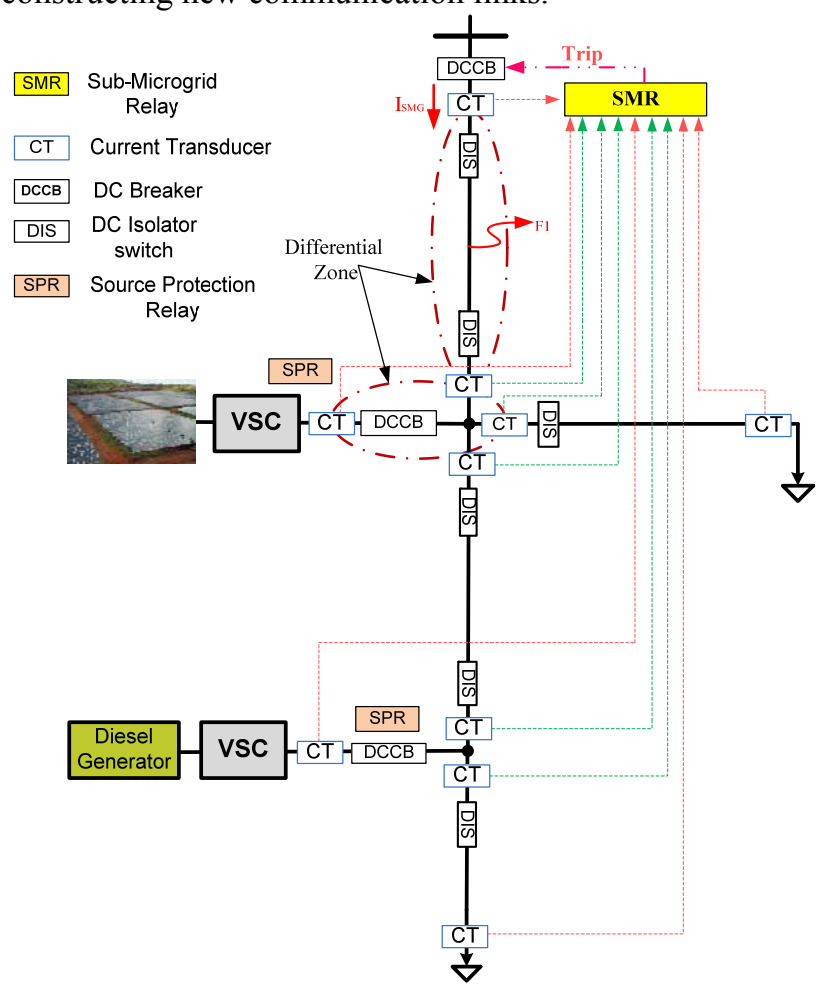

Fig. 2. Required data transmitted for differential and multi-terminal differential protection for a typical SMG.

In the proposed method, each SMG is protected by a submicrogrid relay (SMR) which consists of several differential elements. Each differential element of the SMR receives the measured current at both ends of a dc line and calculates the differential current of the protected line according to (1).

$$
I_{\text {diff }, j}[k]=\left|I_{1, \mathrm{j}}[k]-I_{2, \mathrm{j}}[k]\right|
$$

where $I_{1, \mathrm{j}}$ and $I_{2, \mathrm{j}}$ denote the measured current at both ends of the $j^{t h}$ line, and $k$ is the sample number.

In normal conditions the calculated value for $I_{\text {diff }}$ should be close to zero; however, to prevent the relay's misoperation, the threshold of the differential elements of the SMRs are adjusted considering a restrained current $\left(I_{r}\right)$. In other words, we assume that the $j^{\text {th }}$ differential element of an SMR operates if $I_{\text {diff }, j}[k]>I_{r, j}$. The restrained current of each element can be determined according to the smallest current required for the operation of that element; e.g. $I_{r, j}=K I_{n, j}$. In which $K$ is a reliability coefficient with a value that can be set to $0.1 \ldots 0.25$ and $I_{n, j}$ is the nominal current of the $j^{\text {th }}$ line [18]. After a fault occurrence in the $j^{\text {th }}$ line, the corresponding differential element detects the fault and issues a trip signal. The fact that the difference in (1) is taken in absolute value shows that bi-directional 
power flows caused by DGs cannot impact the performance of the differential scheme.

As shown in Fig. 2, the current measured by the CTs located inside a SMG are transmitted to the corresponding SMR through the communication links. By use of these communication links not only the required data for the differential elements are collected, but also a multi-terminal differential protection can be formed. Indeed, using the measured currents of the SMG's boundaries, transmitted through the red communication links in Fig. 2, the differential current of each SMG can be calculated as shown in (2).

$$
I_{d S M G}[k]=I_{S M G}[k]-\sum_{i=1}^{n-1} I_{b, i}[k]
$$

where $I_{S M G}$ is the sub-microgrid current as shown in Fig. 2, $I_{b, i}$ denotes the measured currents at the SMG's boundaries, and $n$ is the number of the SMGs boundaries.

The trip command of the SMR can be generated by the two-terminal differential as well as by the multi-terminal element. Thus, the SMRs can provide a more stable and reliable protection when they are equipped with this multiterminal differential element.

It is worth noting that one of the issues related to the implementation of the differential protection is that the current of external faults may lead to CT saturation. This issue happens mostly when a high-fault current occurs outside the protection zone of the relay. CT saturation, in turn, leads to the inaccurate measurement of dc currents and consequently results in relay malfunction. In fact, in this case, the relay will operate for faults that occurred outside of its protected zone. Various methods are already presented, as described in [19] to prevent this issue and enhance the performance of the differential protection. However, those methods were presented according to the specifications of current-transformers which are commonly used in ac systems and are not necessarily applicable for the hall-effect current transducers which are used in dc systems. In order to address this issue we introduce an extra unit for the differential elements of the SMRs which can effectively prevent the occurrence of this type of mal-operation. This unit is referred to as restrictive signal generator (RSG).

The RSG unit operates based on the direction of the fault current. It is clear that, only when a fault happens inside a protection zone, the fault current in all the sides of the zone will flow to the inside of the zone. For example, when a fault occurs at the location F1 in Fig. 2, the fault current in both sides of the faulty line will flow from bus to line (fault current goes inside the protection zone). Whereas, when a fault is not inside the protection zone, at least one of the CTs of the zone will detect a fault current that goes to the outside of the zone. Accordingly, when a differential element picks up, the RSG unit receives the direction of the fault current and if the direction of at least one of the currents at the borders of a picked up differential element is to the outside of the zone, it will generate a restrictive signal that prevents the operation of the SMR. Therefore, this restrictive signal improves the performance of the differential unit of SMRs.

The logic circuit of the proposed SMR that can be easily implemented on micro-processor-based relays is shown in
Fig. 3. This figure illustrates that the differential-based unit of SMRs consists of several differential elements. Each of these elements, i.e., Diff_i, protects one of the differential zones of the associated SMG. In addition, the multiterminal differential element is embedded in the differential-based unit that is able to detect faults that occurred inside the SMG. Each of these differential elements is also equipped with an RSG unit to improve its performance. The trip command of the differential-based unit of an SMR is sent to the related DCCB if each of the differential elements detect a fault. Moreover, Fig. 3 shows that the SMR is equipped with overcurrent directional elements as well; these elements operate as a backup unit when the communication link fails. The features and specifications of this backup system are explained in Section IV.B.

It should be noted that SMRs that are located at the borders of two SMGs, are designed to protect both of the SMGs. For example, in Fig. 4, SMR4 receives the measured current from the de lines of SMG2 and SMG4. This SMR sends the trip command to CB9 if it detects a fault in any of these sub-microgrids.

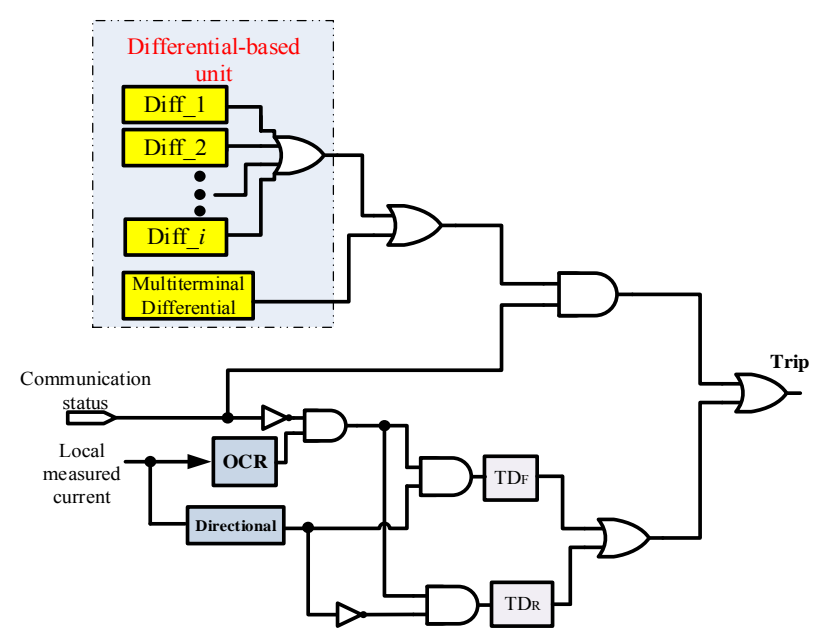

Fig. 3. The logic circuit of the SMRs.

\section{Centralized Protection Unit (CPU)}

The CPU includes two independent units; centralized protection coordinator (CPC) and self-healing and adapting (SHA). The SHA receives the status of the DGs, loads, isolators and CBs. Then it estimates the network topology and calculates new settings for the relays. This unit is also equipped with a self-healing strategy that guarantees the supply-adequacy of on-outage zones of the microgrid. Meanwhile, the CPC supervises the operation of the protection elements of the microgrid and coordinates their operation. All the actions of the protection devices as well as measured currents are reported to both of these units.

\section{The Proposed Protection Scheme}

This section explains the proposed protection strategy, given the elements described in Section III. We address here both the main and the backup protection strategies. The main protection is communication-assisted method; whereas the backup protection is activated when the communication link fails. 


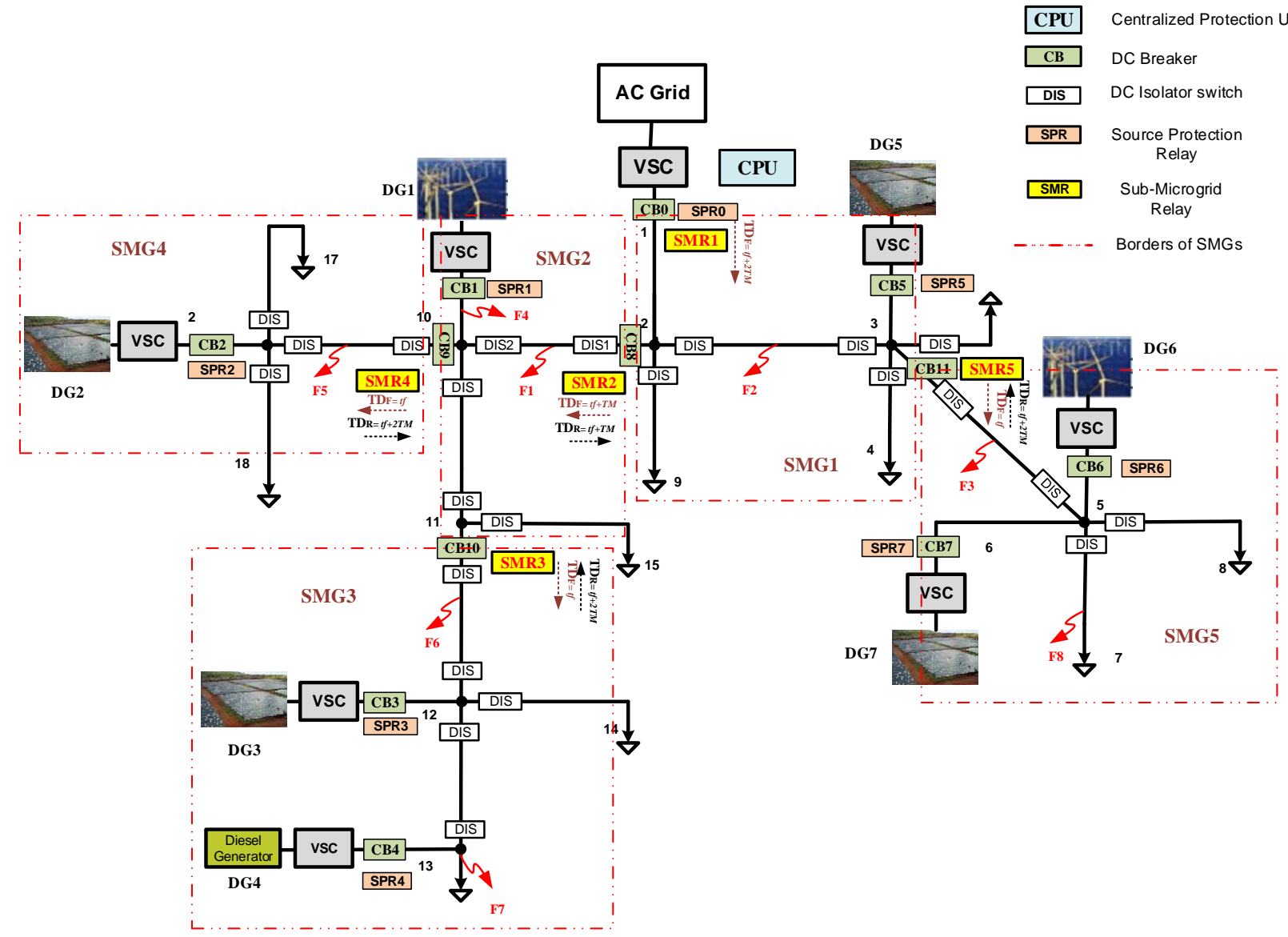

Fig. 4. Study microgid equipped with the protection elements.

\section{A. Main Protection}

Fig. 4 shows the placements of the protection devices presented in Section III into the study microgrid. According to this figure, the following steps are performed after the fault occurrence:

Step 1) the fault is detected by the differential elements of the corresponding SMR. At the same time, the SPRs of the DGs which are inside the faulted sub-microgrid detect the fault. Moreover, according to the type and the location of the fault, the other SPRs which are outside of this submicrogrid may detect the fault as well.

Step 2) the SMR sends the trip signal to the corresponding DCCB. It also reports the fault detection to the CPC by sending an appropriate signal. In this stage, the CPC identifies the faulty sub-microgrid. Simultaneously, the SPRs which detected the fault send the trip signal to the corresponding $\mathrm{CBs}$ if the fault current is not interrupted before the critical time of the related VSC. Since the SMRs use the differential-based protection, they can also detect the HIFs which might not be detectable by SPRs. Therefore, the SMR will send the trip command to the related SPRs as well.

Step 3) the $\operatorname{DCCB}(s)$ that received the trip signal operate and interrupt the fault current. Hence, the faulty submicrogrid is separated from the microgrid. The CBs status then will be reported to the CPU.

Step 4) the open command is sent to the isolators in both ends of the faulted line. Theses isolators are opened when their flowing current decays to zero.
Step 5) after the fault isolation, the opened DCCBs are reclosed by the appropriate commands from the SMRs and the rest of the network is restored.

According to the above process, faults are isolated in Step 4 by opening the isolators at all sides of the faulty zone. Thus, the network restoration of Step 5 is done only when the associated isolators operated successfully. However, isolator failure may impact this operation. For instance, if an isolator in one side of the faulty line fails in opening, then the line is not isolated and hence the fault will remain in the system. To prevent this issue, an isolatorfailure-backup function is introduced for the CPC. Based on this function, when the "open" command is sent to an isolator, the CPC monitors the status of the isolator. If the isolator fails to open within a predetermined time, then the CPC will send the open command to the neighboring isolators.

Based on the requirements of the proposed method, the flowchart of the SPR's operation is shown in Fig. 5.

\section{B. Backup Protection}

Communication-based protection methods are vulnerable to communication failures. Therefore, the proposed method is supported by overcurrent directional elements to back up the differential protection in case the communication network fails. This paper follows the overcurrent directional protection which has been introduced in [14] for active ac microgrids.

The proposed SMRs switch to the overcurrent directional-based backup protection if the communication network fails. It should be noted that, according to the 
specifications of the industrial protocols, the protective devices are equipped with communication failure detection capability and switch to the backup protection without the requirement of any signals from the external supervisory systems [20]. The communication failure detection is handled by exchanging an identification signal between the protection devices. Therefore, if an SMR does not receive the associated identification signals after a predefined period time it will switch to the backup mode, automatically.

As mentioned in Section III.B, based on the location of the DCCBs, the microgrid is divided into several submicrogrids. Each sub-microgrid can be considered as a zone of the backup protection; hence, the directional definitetime overcurrent units are embedded into the SMRs.

As it is shown in Fig. 4, the overcurrent directional element of the SMRs has two operating times for forward and reverse faults, i.e., $T D_{F}$ and $T D_{R}$, respectively. If the communication network fails while a forward fault is detected, a trip command is generated after a time delay $\left(T D_{F}\right)$; while, for the reverse faults, the trip signal is sent to the corresponding $\mathrm{CB}$ after $T D_{R}$.

The operating time of these relays should be determined according to: 1$)$ requirements of the selective protection by relays coordination and 2) the critical time of the main VSC station. Moreover, to provide a selective protection and prevent relay miscoordination, each relay should operate as a backup for its neighboring relay if the fault was not cleared after a time margin (TM). For example, as illustrated in Fig. 4, the maximum operating time of the most upstream relay, i.e., SMR1, is 2TM. Therefore, assuming that the critical time of the main VSC is $T_{C}$, the maximum value of $T M$ is calculated as shown in (3).

$$
T M=\left(T_{C}-\left(t_{C T}+t_{d e t}+t_{C B}\right)\right) / 2
$$

where $t_{C T}$ denotes the delay associated with current transducers and their corresponding A/D converters (several microseconds); $t_{d e t}$ is the fault detection time of the directional overcurrent relay; and $t_{C B}$ denotes the operating time of DCCBs. The operating time of the definite-time overcurrent relays for the backup protection are shown in Fig. 4.

On the other hand, the threshold of the overcurrent element of the $i^{\text {th }} \mathrm{SMR}, I_{S, i}$, is determined according to the nominal current flowing through the corresponding DCCB; i.e., $I_{S, i}=R I_{S M G, i}$, in which $R$ can be set to values ranging from 1 to 1.2 . This current may change after any change in the operational conditions of the microgrid; hence, the relay threshold should be changed by a self-regulation method that is explained in Section V.

The backup strategy, based on directional overcurrent, is implemented as described in the following steps:

Step 0) the SMRs are switched to the backup mode and the CPC is disabled. This step is done once the communication failure is recognized by each relay.

Step 1) the fault is detected by SPRs and SMRs. In this step not only the relays of the faulty zone, but also the relays outside of this zone may detect the fault.

Step 2) the SMRs send the trip signal to the corresponding DCCBs if the fault current was not interrupted before their predetermined time settings. Simultaneously, the SPRs send the trip command if the fault was not cleared before the critical time of the related VSCs.

Step 3) the faulted sub-microgrid is isolated and remains separated from the rest of the microgrid.

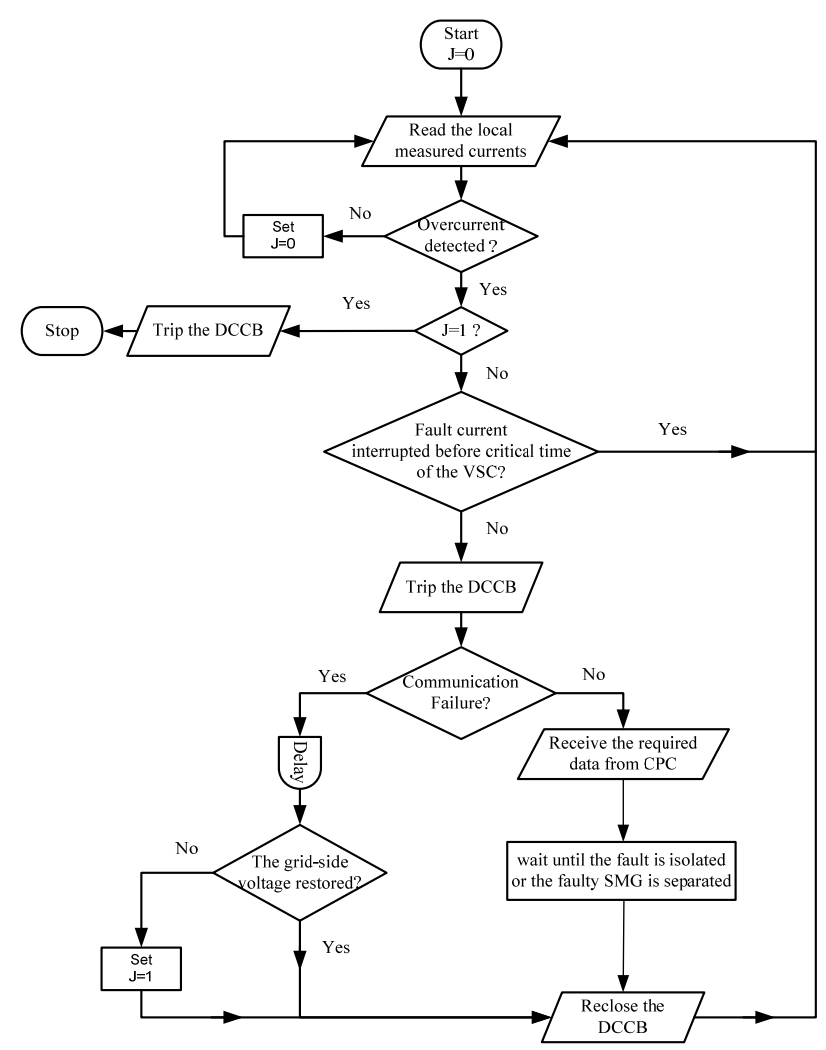

Fig. 5. The proposed algorithm for the SPRs.

Step 4) some of the SPRs may operate even though their VSCs and DG stations are not located inside a faulty submicrogrid. In this step, these stations are reconnected after the fault isolation. The flowchart of the proposed SPRs, displayed in Fig. 5, shows that in the case of communication failure, they will try to reconnect the DGs by use of the following methods:

a) Voltage check: when the DCCB of a DG station is open, the SPR monitors the voltage of the grid-side busbar and reconnects its DG if this voltage returns to an acceptable range ( 0.8 of the nominal voltage is selected in this paper).

b) Auto-reclosing: in some cases it is not possible to detect the fault isolation by monitoring the grid-side voltage. For example, when SMG1 of Fig. 4 is impacted by a fault and isolated, SMG5 will become separated from the rest of the grid as well. Assuming that the protections of all the DG stations inside this SMG disconnect their DGs, the voltage of this grid cannot return to the acceptable range. Consequently, the DGs are not reconnected again and a healthy SMG will remain de-energized. To prevent the occurrence of this problem and to facilitate the network restoring, the autoreclosing ability is embedded into the SPRs. As, shown in Fig. 5 the SPR recloses the corresponding DCCB after a time delay. The DCCBs will remain closed if the faulty sub-microgrid was isolated. 
It should be noted that the above two methods are executed on all the SPRs; hence, the re-closing process will be executed for those DCCBs which are inside the faulted SMG as well. This can cause an automatic networkrestoring, in case of temporary faults, and enhance the reliability.

\section{SELF-HEALING AND ADAPTING UNIT}

Microgrids can operate in grid-connected and islanded modes. Since loads are supplied by the host network and DGs, the load-generation balance is always met in the gridconnected mode. Whereas, in islanded mode, considering the total possible generated power of DGs, additional supervisory actions are needed to achieve the loadgeneration balance. In this case, the supply-adequacy is the main concern about the successful operation of the microgrid. Indeed, supply-adequacy is the initial condition for the reliable operation of the isolated grids. Forming a supply-adequate microgrid by real-time balancing between the load and generation is known as one of the important features of the network self-healing process [21]. Thus, in this paper, a centralized self-healing strategy is presented to guarantee the successful operation of the microgrid in various operational conditions. The same self-healing strategy is used for the on-outage SMGs as well. For example, in Fig. 4 after the isolation of the line between Bus2 and Bus10, i.e., Line210, that has been impacted by F1, SMG2, SMG3 and SMG4 are separated from the host network. In this case, the self-healing process is applied for these SMGs.

Furthermore, as mentioned in Section III.C and Section IV.B, the thresholds of both the main and backup elements of the SMRs are determined according to the nominal currents of the protected lines of the associated SMGs. The nominal current of the lines may vary due to the changes in the microgrid operation modes, load variations or the intermittent behavior of the DGs. Thus, using the fixed relay settings may lead to protection issues such as relays mal-operation and protection blinding [8]. For this reason, in this paper the thresholds of the SMRs are updated after significant changes in the pre-noted factors.

Briefly, to deal with the above mentioned issues, the microgrid is equipped with the centralized self-healing and adapting (SHA) unit that has the following main tasks:

a) Providing network self-healing to guarantee the supply-adequacy when 1) the microgrid works in the islanding mode; and/or 2) SMGs are separated from the grid.

b) Adapting the SMRs settings according to the network operational conditions.

These goals are achieved by use of the following steps:

Step 1) In the first step, the SHA collects the required information from the isolated microgrid or separated zone. The most important information is the power of the connected loads, output power of the DGs and the free capacity of the dispatchable DGs (DDGs) [21]. According to this information, the SHA determines the value of the power which should be disconnected from the on-outage zone. Then, the candidate loads which should be shed are selected based on the pre-determined load priority.
Step 2) The appropriate signals are sent to the loads that are selected in Step 1.

Step 3) The DDGs are re-dispatched and the settings of the SPRs are adapted based on the new output current of the corresponding VSCs.

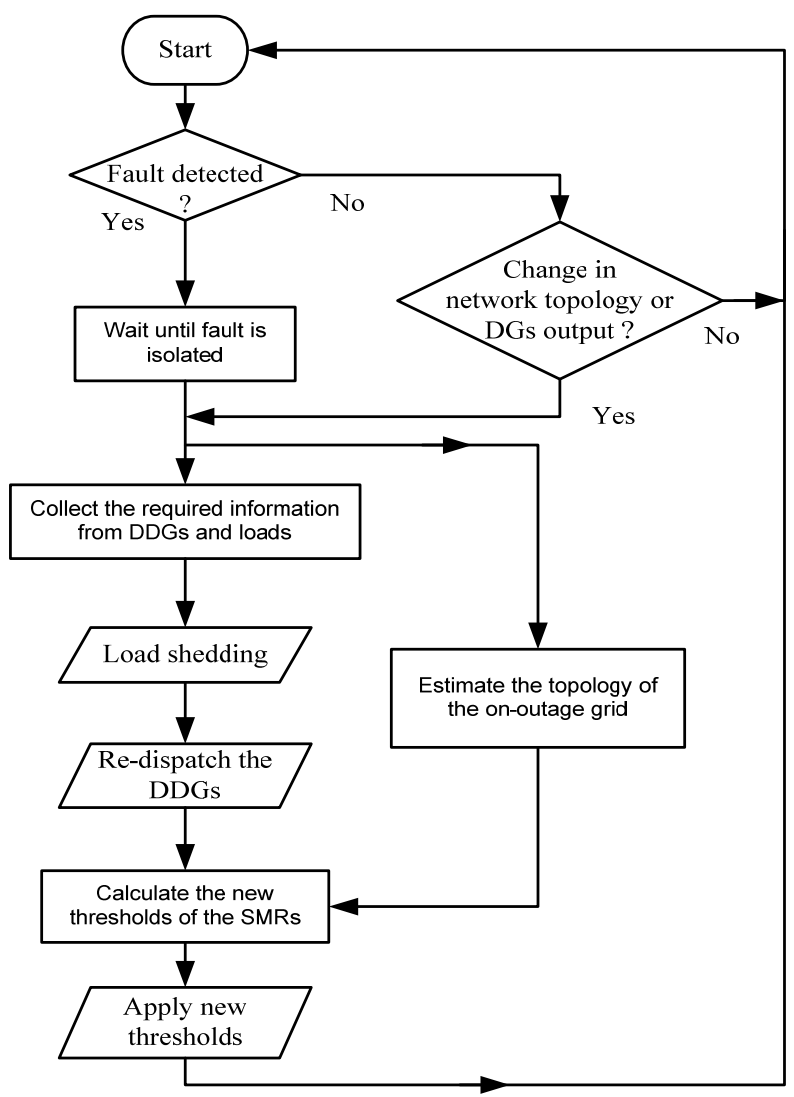

Fig. 6. The proposed algorithm of the SHA.

Step 4) Considering the status of the isolators and DCCBs, the topology of the on-outage zone is estimated (See Section V.A).

Step 5) New thresholds for the SMRs are calculated according to the load flow equations in dc grids (the method is explained below in Section V.A).

Step 6) The new settings are applied to SPRs and SMRs.

\section{A. Re-calculating the SMRs Setting}

The SHA calculates the new threshold of the SMRs based on the power flow equations of the dc systems presented in [22]. In a dc network, the power flowing from the dc buses to the dc grid is given by:

$$
\mathbf{P}=\mathbf{U} \otimes(\mathbf{Y U})
$$

where the vector $\mathbf{U}$ denotes the dc bus voltages, $\mathbf{Y}$ denotes the admittance matrix of the dc grid, and $\otimes$ is the Hadamard product operator. Vectors $\mathbf{P}$ and $\mathbf{U}$ are introduced in (5).

$$
\mathbf{P}=\left[\begin{array}{c}
\mathrm{P}_{1} \\
\cdot \\
\cdot \\
\cdot \\
\mathrm{P}_{\mathrm{n}}
\end{array}\right], \quad \mathbf{U}=\left[\begin{array}{c}
\mathrm{U}_{1} \\
\cdot \\
\cdot \\
\cdot \\
\mathrm{U}_{\mathrm{n}}
\end{array}\right]
$$

After a change in the injected power at the dc busses, the 
dc voltage variation of the network can be obtained by using (6).

$$
\Delta \mathbf{U}=\mathrm{J}_{\mathrm{DC}}^{-1} \Delta \mathbf{P}
$$

where $\Delta \mathbf{P}$ denotes the nodal power variation and $\mathrm{J}_{\mathrm{DC}}$ is the Jacobian matrix of the dc grid that can be calculated directly by using (7).

$$
\mathbf{J}_{\mathbf{D C}}=\operatorname{diag}(\mathbf{U}) \cdot \mathbf{Y}+\operatorname{diag}(\mathbf{Y U})
$$

where diag refers to the mathematical operator which converts a vector into a diagonal matrix.

Substituting (7) into (6), the voltages of the DC busses after a change in the nodal injected power are calculated as shown in (8).

$$
\mathbf{U}_{2}=\mathbf{U}_{1}+\mathbf{J}_{\mathbf{D C}}^{-\mathbf{1}} \Delta \mathbf{P}
$$

Here, vectors $\mathbf{U}_{\mathbf{1}}$ and $\mathbf{U}_{\mathbf{2}}$ denote the voltages of dc busses before and after an event, respectively. Finally, the current of each dc feeder could be found by using (9).

$$
\mathbf{I}=\mathbf{Y} \cdot \mathbf{U}_{2}=\mathbf{Y} \cdot\left(\mathbf{U}_{1}+\mathbf{J}_{\mathrm{DC}}^{-1} \Delta \mathbf{P}\right)
$$

The equation (9) shows that any change in the network topology, reflected in the $\mathbf{Y}$ matrix, may impact the direction and/or the magnitude of the distribution line current. Furthermore, this equation illustrates that the connection/disconnection of DGs or changes in their generated power, reflected in $\Delta \mathbf{P}$, may also result in significant changes in the feeders' currents.

Thus, in order to calculate the new pickup currents, the SHA monitors: 1) the status of the DCCBs and isolators to estimate the network topology and to update the $\mathbf{Y}$ matrix; 2) the injected power of the DGs and the load currents; and 3 ) the voltage of the dc busses. Then, the SHA calculates the new pickup currents and communicates with the SMRs to apply the new settings. Fig. 6 shows the flowchart of the proposed self-heling strategy to be implemented in the SHA unit.

\section{HIL VERIFICATION}

To validate the proposed protection scheme in the context of the network shown in Fig. 4, the Hardware-In-the Loop (HIL) simulation approach was used. The HIL method was introduced to investigate errors and delays that do not appear in the classical off-line simulations. Fig. 7 shows the schematic diagram of the HIL setup. This setup consists of: 1) OPAL-RT as a real time simulator which simulates the microgrid of Fig. 4 ; 2) a PC as the command station (programming host) that is used to run the Matlab/Simulink model that will be executed on the OPAL-RT; 3) a development board (DK60 from Beck.) that is used to implement the elements of the proposed protection scheme; and 4) a router that is used to connect all the setup devices in the same sub-network. The OPAL-RT is also connected to the DK60 board through Ethernet ports. More details about the components of this setup are introduced in the previous work of the authors in [23].

\section{CASe Studies}

In the following paragraphs, several fault scenarios are simulated to demonstrate the effectiveness of the proposed method. In all the study cases, it is assumed that a fault is initiated at $\mathrm{t}=1.0 \mathrm{~s}$. Also, it is assumed that the operating time of the medium voltage $\mathrm{AC} \mathrm{CBs,} \mathrm{which} \mathrm{are} \mathrm{used} \mathrm{here}$ as the isolator switches, is around $55-60 \mathrm{~ms}$. Moreover, the operating time of the solid-state DCCBs is assumed to be less than $1 \mathrm{~ms}[15]$.

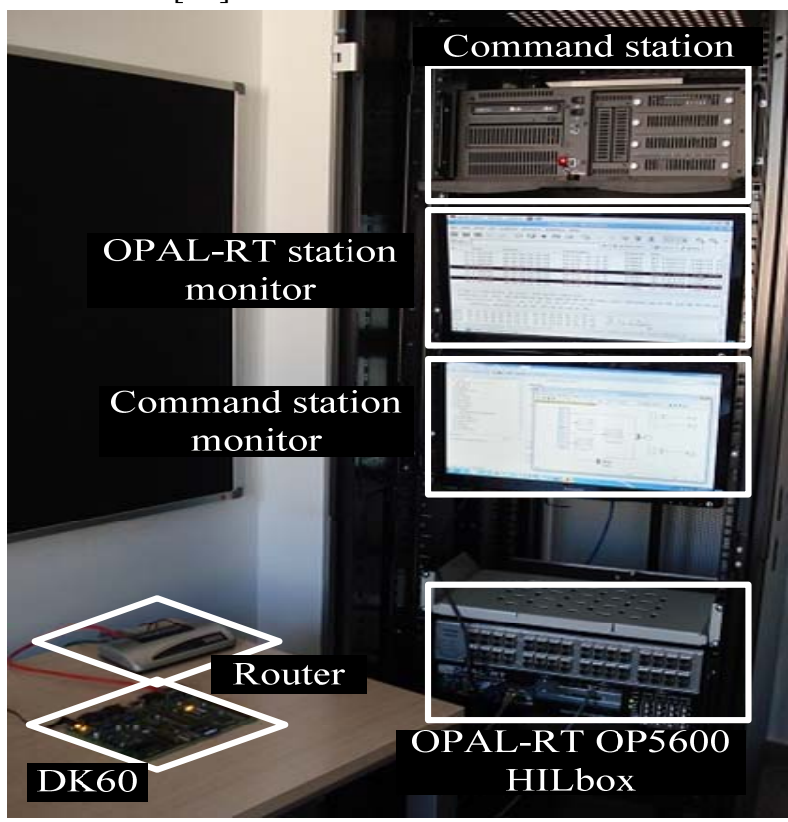

Fig. 7. The schematic diagram of the HIL setup.

\section{A.Case Study 1: Operation of the main protection}

In the first case study we assumed that a solid pole to pole (PP) fault impacts the microgrid at the point labeled with F1 in Fig. 4. Subsequent to the fault occurrence, SMR2, SMR3 and SMR4 detect the fault and send the trip command to the corresponding DCCBs after $2.4 \mathrm{~ms}( \pm 0.2 \mathrm{~ms})$. $\pm 0.2 \mathrm{~ms}$ is the difference between the operating times of these SMRs. The corresponding DCCBs operate and isolate SMG2 around $3.6 \mathrm{~ms}$ after the fault occurrence. Meanwhile, SPR1 detects the fault $48 \mu \mathrm{s}$ after its occurrence; however, since its operating time has been set according to the critical time of the VSC of DG1, it will send the trip command after $3.2 \mathrm{~ms}$.

Simultaneously, the relays of the DGs outside the faulty SMG that the critical time of their VSC station is less than the fault clearing time will operate as well. For example, the operating time of SPR2 is set to $2.7 \mathrm{~ms}$ while the fault current fed from the SMG4 is interrupted after $3.6 \mathrm{~ms}$; hence, SPR2 sends the trip command to DCCB2 and isolates DG2. This DG should be re-connected as soon as possible. All of these operations and commands are reported to the CPC. When the CPC receives the "open" status of the DCCBs, it will send two different commands. First, the "reclose" command is sent to SPR2 which is not inside the faulty SMG. Second, after a time margin, which we set to $10 \mathrm{~ms}$, the CPC sends the "open" command to the corresponding isolator switches. Therefore, considering the operating time of these switches, the faulty line is isolated in less than $80 \mathrm{~ms}$ after the fault occurrence. The open status of these switches is reported to the CPC and considering the time margin for reliable operation the "close" command is sent to SMR2, SMR3, SMR4 and SPR1 93ms after the fault occurrence. The operating time of the various parts of the proposed protection technique is shown in Fig.8. This test illustrates that the proposed protection is able to isolate the faulty part and restore the sound parts of the microgrid in 
less than $100 \mathrm{~ms}$.

This case study also illustrates that by the use of a minimum number of DCCBs, the method provides a selective protection with the minimum possible interrupted loads and very short interruption duration. In other words, the minimum possible loads are disconnected and the rest of the faulty sub-microgrid is restored after around $100 \mathrm{~ms}$. This behavior enhances the network reliability, and due to the direct relation between the reliability indexes and the economic aspects of the network operation [24, 25], the method reduces the outage costs as well.

\section{Case Study 2: Operation of the backup protection when communication fails}

After a communication failure, the SMRs switch to their backup mode. In this case, as explained in Section IV.B, the $T D_{F}$ and $T D_{R}$ of each SMR are set according to the $T M$. The method for calculating the TM was explained in Section IV.B. The simulation results show that the critical time of the main VSC station is $8.5 \mathrm{~ms}$; then, according to (2) the maximum value of $T M$ is $3.5 \mathrm{~ms}$; however, $T M$ is set to $2.5 \mathrm{~ms}$.

Now, let's assume that a solid pole to ground (PG) fault occurs at the point labeled with F3 in Fig. 4 while the communication link fails. In this case, SPR5, SPR6, SPR7, and SMR5 detect the fault and SMR5 sends the trip command immediately. Consequently, SMG5 is isolated from the rest of the network after $1.1 \mathrm{~ms}$. Furthermore, according to the critical time of the corresponding VSCs, SPR5, SPR6 and SPR7 are going to send the trip command after $2.8 \mathrm{~ms}, 2.5 \mathrm{~ms}$, and $3.1 \mathrm{~ms}$, respectively. However, due to the separation of the SMG5 before the operating time of SPR5, this relay will not send its trip command. By the operation of CB6 and CB7, DG6 and DG7 are disconnected in around $4 \mathrm{~ms}$ after the fault occurrence. It is clear that, although this backup method can provide a high-speed fault interruption, the faulty SMG will be separated after any fault occurrence. As it is shown in Fig. 9, to facilitate the fast network restoring, all the relays which have been sent the trip command will send the reclosing command after a time delay. For our study case we set this time delay to be $0.3 \mathrm{~s}$. Hence, in the case of a temporary fault occurrence, the isolated SMG may restore after around $0.3 \mathrm{~s}$.

To further evaluate the effectiveness of the proposed protection strategy, in addition to the above two cases, various fault scenarios have also been studied, however, due to the space limitation, the results of only a selected number of test cases have been reported in Table 1. It should be noted that for each case, only the operating time of the relays inside the faulty SMG is reported in Table 1.

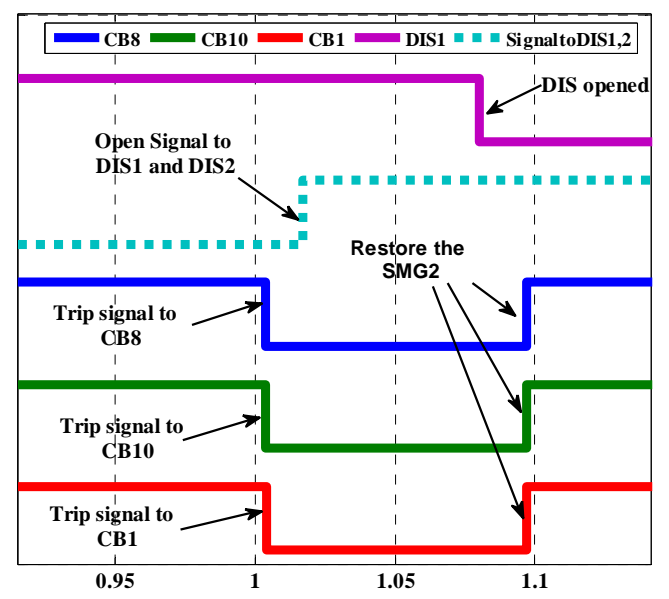

Fig. 8. The operation time of the protection elements for case study1.

Table 1. Operating times of the main and backup protection for selected fault scenarios.

\begin{tabular}{|c|c|c|c|c|c|c|c|}
\hline \multirow{2}{*}{$\begin{array}{l}\text { Fault } \\
\text { Type }\end{array}$} & \multirow{2}{*}{$\begin{array}{c}\text { Fault } \\
\text { Location }\end{array}$} & \multicolumn{3}{|c|}{ Main Protection } & \multicolumn{3}{|c|}{$\begin{array}{c}\text { Backup Protection } \\
\text { (Communication Failure) }\end{array}$} \\
\hline & & $\begin{array}{l}\text { Operated } \\
\text { Relay(s) }\end{array}$ & $\begin{array}{l}\text { Operating } \\
\text { Time (ms) }\end{array}$ & $\begin{array}{c}\text { Faulty SMG } \\
\text { Restored After } \\
\text { (ms) }\end{array}$ & $\begin{array}{l}\text { Operated } \\
\text { Relay(s) }\end{array}$ & $\begin{array}{l}\text { Operating } \\
\text { Time (ms) }\end{array}$ & $\begin{array}{c}\text { For Temporary Faults, } \\
\text { Faulty SMG Restored } \\
\text { After (ms) }\end{array}$ \\
\hline \multirow[t]{6}{*}{ PP } & F2 & SMR1 & 2.5 & 97 & SMR1 & 5 & 307 \\
\hline & & SMR5 & 2.6 & & SMR5 & 5.2 & \\
\hline & & SPR5 & 2.8 & & SPR5 & 2.8 & \\
\hline & F7 & SMR3 & 2.4 & 99 & SMR3 & 0.12 & 306 \\
\hline & & SPR3 & 3.8 & & SPR3 & 3.8 & \\
\hline & & SPR4 & 4.2 & & SPR4 & 4.2 & \\
\hline \multirow[t]{8}{*}{ PG } & F4 & SMR2 & 2.7 & 98 & SMR2 & 2.52 & 307 \\
\hline & & SMR3 & 2.6 & & SMR3 & 5.3 & \\
\hline & & SMR4 & 2.5 & & SMR4 & 5.35 & \\
\hline & & SPR1 & 3.2 & & SPR1 & 3.2 & \\
\hline & F5 & SMR4 & 2.6 & 97 & SMR4 & 0.21 & 305 \\
\hline & & SPR2 & 2.7 & & SPR2 & 2.7 & \\
\hline & F6 & SMR3 & 2.8 & 99 & SMR3 & 0.25 & 308 \\
\hline & & SPR3 & 3.8 & & SPR3 & 3.8 & \\
\hline
\end{tabular}




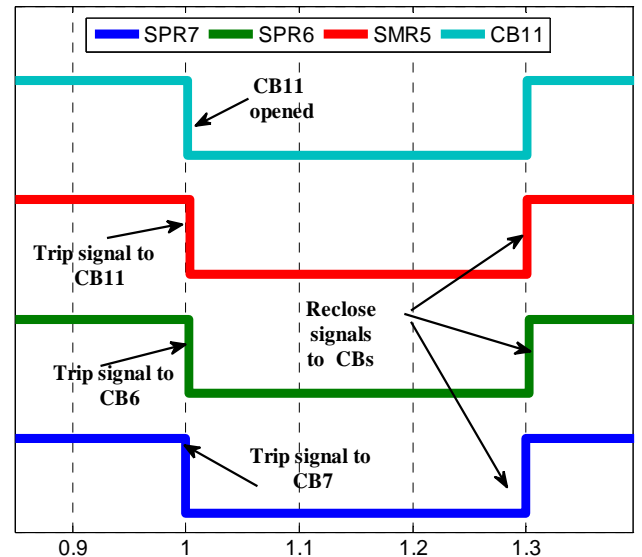

Fig. 9. The operation time of the protection elements for case study2.

\section{CONCLUSIONS}

This paper proposes a centralized protection strategy for VSC-based dc microgrids. The proposed strategy also presents a communication-assisted method which is able to detect the faulty zone without using complex calculations. The HIL simulation results show that the proposed scheme is fast enough to guarantee the safety of the VSCs that supply the dc buses and of the other important elements of the system. The results also show that the proposed strategy restores the network within $100 \mathrm{~ms}-300 \mathrm{~ms}$.

Since the use of DCCBs for all the dc lines is not economically feasible for most microgrids and distribution systems, one of the main advantages of this method is that it provides a selective protection strategy by making use of a minimum number of DCCBs. The method is also complemented with a self-healing strategy which can facilitate the network-restoration. The self-heling strategy guarantees that each sub-microgrid continues its normal operation even if it is isolated from the main source. Moreover, in order to provide an effective network restoration, it is necessary to use a fast and selective fault location method. Thus, the proposed method has been equipped with a differential-based protection that can be implemented on the communication infrastructure of the smart grid.

From the economical point of view, not only the selective protection is achieved with a minimum number of DCCBs, but also, due to the fast network-restoration, the method reduces the interrupted zone and interruption time which are reflected in the reduction of outage costs.

\section{REFERENCES}

[1] E. Sortomme, S. Venkata, and J. Mitra, "Microgrid protection using communication-assisted digital relays," IEEE Trans. Power Del., vol. 25 , no. 4, pp. 2789-2796, 2010.

[2] D. Salomonsson, L. Soder, and A. Sannino, "Protection of lowvoltage DC microgrids," IEEE Trans. Power Del., vol. 24, no. 3, pp. 1045-1053, 2009.

[3] T. Hakala, T. Lähdeaho, and P. Järventausta, "Low-Voltage DC Distribution-Utilization Potential in a Large Distribution Network Company," IEEE Trans. Power Del., vol. 30, no. 4, pp. 1694-1701, 2015.
[4] J. Yang, J. E. Fletcher, and J. O'Reilly, "Short-circuit and ground fault analyses and location in VSC-based DC network cables," IEEE Trans. Ind. Elec., vol. 59, no. 10, pp. 3827-3837, 2012.

[5] C. Gavriluta, J. I. Candela, J. Rocabert, A. Luna, and P. Rodriguez, "Adaptive Droop for Control of Multiterminal DC Bus Integrating Energy Storage," IEEE Trans. Power Del., vol. 30, no. 1, pp. 16-24, 2015.

[6] P. Rakhra, P. Norman, S. Fletcher, S. Galloway, and G. Burt, "Evaluation of the Impact of High-Bandwidth Energy-Storage Systems on DC Protection," IEEE Trans. Power Del., vol.30, pp. 1-1, 2015.

[7] S. D. A. Fletcher, P. J. Norman, S. J. Galloway, P. Crolla, and G. M. Burt, "Optimizing the Roles of Unit and Non-unit Protection Methods Within DC Microgrids," IEEE Trans. Smart Grid, vol. 3, no. 4, pp. 2079-2087, Dec 2012.

[8] M. Monadi, M. A. Zamani, A. Luna, J. I. Candela, and P. Rodriguez, "Protection of AC and DC distribution systems Embedding distributed energy resources: A comparative review and analysis," Renew. Sust. Energy Rev., vol. 51, pp. 1578-1593, 2015.

[9] M. E. Baran and N. R. Mahajan, "Overcurrent protection on voltagesource-converter-based multiterminal DC distribution systems," IEEE Trans. Power Del., vol. 22, no. 1, pp. 406-412, 2007.

[10] W. Leterme, J. Beerten, and D. Van Hertem, "Non-unit protection of HVDC grids with inductive dc cable termination," IEEE Trans. Power Del., vol. 30, pp. 1-1, 2015.

[11] W. L. Li, A. Monti, and F. Ponci, "Fault Detection and Classification in Medium Voltage DC Shipboard Power Systems With Wavelets and Artificial Neural Networks," IEEE Trans. Instr. Meas., vol. 63, no. 11 , pp. 2651-2665, Nov 2014.

[12] J. D. Park, J. Candelaria, L. Y. Ma, and K. Dunn, "DC Ring-Bus Microgrid Fault Protection and Identification of Fault Location," IEEE Trans. Power Del., vol. 28, pp. 2574-2584, Oct 2013.

[13] S. D. A. Fletcher, P. J. Norman, K. Fong, S. J. Galloway, and G. M. Burt, "High-Speed Differential Protection for Smart DC Distribution Systems," IEEE Trans. Smart Grid, vol. 5, no. 5, pp. 2610-2617, Sep 2014.

[14] M. A. Zamani, A. Yazdani, and T. S. Sidhu, "A communicationassisted protection strategy for inverter-based medium-voltage microgrids," IEEE Trans. Smart Grid, vol. 3, no. 4, pp. 2088-2099, 2012.

[15] C. M. Franck, "HVDC Circuit Breakers: A Review Identifying Future Research Needs," IEEE Trans. Power Del., vol. 26, no. 2, pp. 998-1007, 2011.

[16] M. K. Bucher and C. M. Franck, "Fault Current Interruption in Multiterminal HVDC Networks," IEEE Trans. Power Del., vol. 31, no. 1 , pp. 87-95, 2016.

[17] S. A. Arefifar, Y. A. R. I. Mohamed, and T. H. M. El-Fouly, "Comprehensive Operational Planning Framework for Self-Healing Control Actions in Smart Distribution Grids," IEEE Trans. Power Syst., vol. 28, no. 4, pp. 4192-4200, 2013.

[18] P. M. Anderson, Power system protection vol. 1307: McGraw-Hill New York, 1999.

[19] M. Stanbury and Z. Djekic, "The Impact of Current-Transformer Saturation on Transformer Differential Protection," IEEE Trans. Power Del., vol. 30, no. 3, pp. 1278-1287, 2015.

[20] "IEC 61850-8-1, Communication networks and systems in substations - Part 8-1: Specific Communication Service Mapping (SCSM) - Mappings to MMS (ISO 9506-1 and ISO 9506-2) and to ISO/IEC 8802-3," ed: IEC, 2004.

[21] Z. Wang and J. Wang, "Self-Healing Resilient Distribution Systems Based on Sectionalization Into Microgrids," IEEE Trans. Power Syst., vol. 30, pp. 1-11, 2015.

[22] T. M. Haileselassie and K. Uhlen, "Impact of DC Line Voltage Drops on Power Flow of MTDC Using Droop Control,", IEEE Trans. Power Syst., vol. 27, no. 3, pp. 1441-1449, 2012.

[23] M. Monadi, C. Koch-Ciobotaru, A. Luna, J. I. Candela, and P. Rodriguez, "A protection strategy for fault detection and location for multi-terminal MVDC distribution systems with renewable energy systems," in Proc IEEE Rene. Energy Rese. App., pp. 496-501. Oct. 2014.

[24] J. F. Prada, "The Value of Reliability in Power Systems - Pricing Operating Reserves -," Energy Laboratory, Massachusetts Institute of Technology, 1999.

[25] R. F. Ghajar and R. Billinton, "Economic costs of power interruptions: a consistent model and methodology," Inter. Jour. Elect. Power Energy Syst., vol. 28, no. 1, pp. 29-35, 2006. 


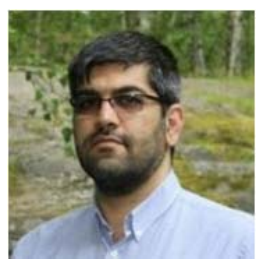

Mehdi Monadi (M'16) received the B.Sc. degree in electrical engineering from the Shahid Chamran University of Ahvaz, Ahvaz, Iran, in 2001 and the M.Sc. degree in electrical engineering from the Shahrood University of Technology, Shahrood, Iran, in 2004.

Currently, he is a Ph.D student in Research Center on Renewable Electrical Energy Systems (SEER) in the Department of Electrical Engineering of the Technical University of Catalonia (UPC), Barcelona, Spain. In 2015 he was a visiting $\mathrm{PhD}$ student in the SmarTs Lab in the Royal Institute of Technology (KTH), Stockholm, Sweden.

His research interests are protection of active distribution system, and dc distribution and transmission systems.

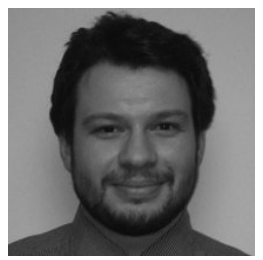

Catalin Gavriluta ( $\left.\mathrm{S}^{\prime} 13\right)$ was born in Arad, Romania, in 1986. He received the engineering degree in automation and applied informatics from the Polytechnic University of Timisoara, Timisoara, Romania, and the M.Sc. degree in wind power systems from Aalborg University, Aalborg, Denmark, in 2011, respectively. In 2015 he received a Ph.D. degree in electrical engineering from the Technical University of Catalonia, Barcelona, Spain. His research interests were about the control and operation of multiterminal dc networks used for the large-scale integration of solar power supported by energy storage. He is currently having a research position within Grenoble INP-Univ. Grenoble Alps, France, on distributed control and interactions between Green ICTs and Smart Grids.

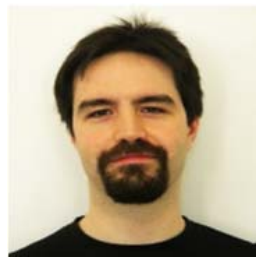

Alvaro Luna (S'07-M'10) received the B.Sc., M.Sc., and Ph.D. degrees in electrical engineering from the Technical University of Catalunya (UPC), Barcelona, Spain, in 2001, 2005, and 2009, respectively.

He joined the faculty of UPC in 2005, where he is currently an Assistant Professor. His research in terests include wind turbines control, integration of distributed generation, and power conditioning.

Dr. Luna is a member of the IEEE Power Electronics Society, the IEEE Industrial Electronics Society, and the IEEE Industrial Applications Society.

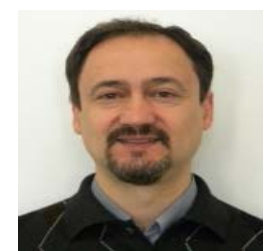

J. Ignacio Candela ( $\left.\mathrm{S}^{\prime} 99-\mathrm{M}^{\prime} 04\right)$ received the B.S. and M.S. degrees in industrial engineering and the $\mathrm{Ph} . \mathrm{D}$. degree in electrical engineering from the Technical University of Catalunya (UPC), Barcelona, Spain, in 1987, 2000, and 2009, respectively. In 1990, he became an Assistant Professor at UPC, where he later advanced to Associate Professor in 1993. Currently, he is part of the research group on Renewable Electrical Energy Systems, Department of Electrical Engineering. He has authored or co-authored more than 30 published technical papers, and holds several patents. His current research interests include power conditioning, integration of distributed energy systems, and the control of grid-connected power converters.

Dr. Candela is a member of the IEEE Power Electronics Society, the IEEE Industrial Electronics Society, and the IEEE Industry Application Society.

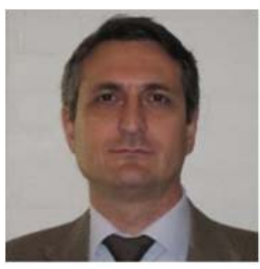

Pedro Rodriguez (SM'10-F'13) received the M.Sc. and Ph.D. degrees in electrical engineering from the Technical University of Catalonia (UPC), Barcelona, Spain, in 1994 and 2004, respectively. He was a PostDoctoral Researcher at the Center for Power Electronics Systems, Virginia Tech, USA, in 2005, and at the Department of Energy Technology, Aalborg University (AAU), Denmark, in 2006. He joined the faculty of UPC as an Assistant Professor in 1990, where he became the Director of the Research Center on Renewable Electrical Energy Systems, Department of Electrical Engineering. He is currently a part time Professor with UPC. He was also a Visiting Professor at AAU from 2007 to 2011, and a Co-Supervisor of the Vestas Power Program. In 2011, he joined Abengoa, Seville, Spain, where he is currently the Director of Technology on Power Systems and Power Electronics. He has coauthored one book, over 70 papers in ISI technical journals and around 250 papers in conference proceedings. He is the holder of twelve licensed patents. He received the Best Technical Letter Award 2012 and the Second Best Paper Award 2012 in the IEEE Transactions on Power Electronics and the Second Best Paper Award 2014 in the IEEE Journal of Emerging and Selected Topics in Power Electronics. His current research interests include distributed power systems, flexible transmission systems, and power conversion.

Dr. Rodriguez is an IEEE Fellow for his contributions in the control of distributed generation, an Associate Editor of the IEEE TRANSACTION ON POWER ELECTRONICS. He was the Vice Chair of the Sustainability and Renewable Energy Committee of the IEEE Industry Application Society. 